\title{
CARACTERIZAÇÃO MECÂNICA E ANÁLISE DE FALHAS DE CHAPAS FABRICADAS PELO PROCESSO DE TAILOR WELDED BLANK SUBMETIDAS A ESTAMPAGEM PROFUNDA*
}

\author{
Etiene Pereira de Andrade 1 \\ Wellington Augusto dos Santos ${ }^{2}$ \\ Alexandre Queiroz Bracarense ${ }^{3}$
}

\section{Resumo}

Em decorrência de índices de emissão de poluentes cada vez mais restritivos, de programas de incentivo à eficiência energética e do uso de fontes de energia renováveis, a indústria automobilística se renova e desenvolve tecnologias que permitam redução de massa dos veículos conciliada a bom desempenho estrutural e em serviço. Nesse contexto pode-se destacar o processo de Tailor Welded Blank (TWB). Esse processo permite que se reduza o número de reforços empregados no veículo, bem como a necessidade de um maior número de etapas de manufatura. Este estudo trata da caracterização das fraturas observadas em chapas obtidas pelo processo de TWB, para aplicação em portas laterais de veículos, quando submetidas à operação de estampagem profunda. Para a identificação da causa raiz foram realizados os ensaios de tração (de forma qualitativa, buscando apenas definir a região de falha), embutimento e análises metalográficas usando microscópio óptico (MO) microscópio eletrônico de varredura por feixe de elétrons (MEV). Os resultados indicaram que a fratura ocorreu na zona termicamente afetada, e não na zona fundida. Verificou-se, ainda, que a fratura ocorreu por esforços de tração simples, gerados pelo processo de estampagem do componente.

Palavras-chave: Conformação; Soldagem a laser; Talior Welded Blank; Indústria automobilística.

\section{MECHANICAL CHARACTERIZATION AND FAILURE ANALYSIS OF PLATES MANUFACTURED BY THE TAILOR WELDED BLANK PROCESS SUBMITTED TO DEEP DRAWING}

\section{Abstract}

As a result of ever more restrictive pollutant emission rates, programs to encourage energy efficiency and the use of renewable energy sources, the automotive industry is renewing and developing technologies that allow the reduction of mass of vehicles, which is reconciled with good structural and in service performance. In this context the Tailor Welded Blank (TWB) process can be highlighted. This process allows reducing the number of reinforcements used in the vehicle, as well as the need for a greater number of manufacturing steps. This study deals with the characterization of the fractures observed in plates obtained by the TWB process, for application in side doors of vehicles, when subjected to the deep drawing operation. In order to identify the root cause, tensile tests were performed (in a qualitative way, seeking only to define the region of failure), inlay and metallographic analyzes using optical microscopy (OM) and electron beam scanning electron microscope (SEM). The results indicated that the fracture occurred in the thermally affected zone, not in the molten zone. It was also verified that the fracture occurred by simple tensile forces generated by the stamping process of the component.

Keywords: Forming; Laser beam welding; Tailor Welded Blank; Automobile industry.

1 Engenharia de Materiais, Mestrando em Processos de Fabricação, Universidade Federal de Minas Gerais, Belo Horizonte - MG, Brasil, andrade.etiene@hotmail.com.

2 Engenheiro Mecânico, Analista de Manufatura Pleno, Engenharia de Manufatura de Estampos, FIAT Chrysler Automóveis do Brasil LTDA, Betim, MG, Brasil, Wellington.santos@fcagroup.com..

3 Físico, Professor, Pesquisador, PhD - Universidade Federal de Minas Gerais (UFMG), Departamento de Engenharia Mecânica, Belo Horizonte, Minas Gerais, Brasil., bracarense@ufmg.br. 


\section{INTRODUÇÃO}

O processo de fabricação por Tailor Blank consiste basicamente em soldar blanks semiacabados, compostos por ao menos duas chapas distintas (em termos de material, espessura, revestimentos e outras propriedades física e/ou químicas), antes da etapa de conformação final para obtenção do produto acabado. A solda normalmente é feita a laser, principalmente quando se trabalha com produção em série. Destaca-se que a região soldada não precisa ser necessariamente linear, permitindo o trabalho com peças das mais diversificadas geometrias [1,2,3]. Tem-se, segundo Marlein et al [1], conforme se apresenta na Figura 1, quatro processos principais de fabricação de Tailor Blanks:

- $\quad$ Patchwork Blanks: Duas chapas distintas, soldadas de maneira sobreposta uma à outra.

- Tailor Welded Blanks: Duas ou mais chapas, de materiais similares ou completamente diferentes (em termos de espessura ou propriedades físicas e químicas) são soldadas, no mesmo plano, formando um blank único.

- Tailor Rolled Blank: Uma mesma chapa é submetida, em diferentes regiões, a esforços distintos de laminação, conferindo-lhe regiões com espessuras distintas, mas mesmo material.

- $\quad$ Tailor Heat Treated Blanks: Consiste em tratar termicamente os blanks obtidos com intuito de melhorar sua conformabilidade, otimizando a obtenção do produto final.

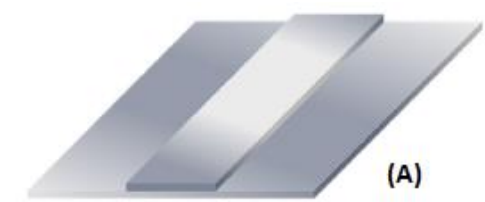

(A)

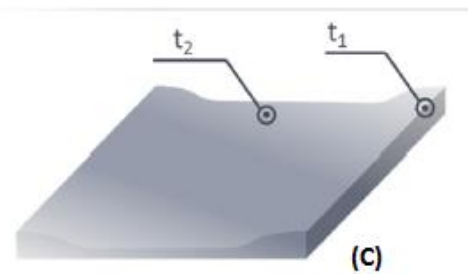

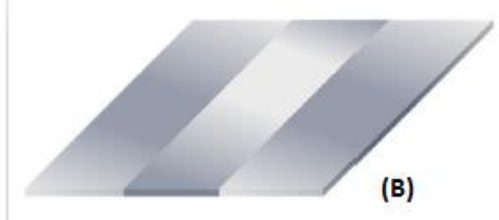

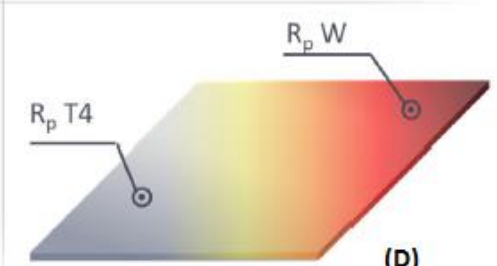

(D)

Figura 1 - Diferentes processos de fabricação de Tailor Blanks. (A) Patchwork Blanks; (B) Tailor Welded Blanks; (C) Tailor Rolled Blank e (D) Tailor Heat Treated Blanks. (Fonte: Marklein et al, 2014).

Uma das principais aplicações da referida tecnologia é a confecção de peças para empresas automobilísticas. De modo geral, cada componente requerido para compor o automóvel é produzido de forma separada e, em regiões de maior solicitação mecânica é usual o uso de reforços, soldados um a um também. Além do grande número de operações de manufatura, gera-se gradativo aumento de massa nos componentes à medida que se demanda maior resistência mecânica das peças confeccionadas [4].

Por meio do processo de Tailor Welded Blank (TWB) consegue-se confeccionar peças com espessura diferenciada na região de maior solicitação, dispensando 0 uso de reforços e componentes adicionais. Porém, tem-se a dificuldade de se manter a espessura do produto conformado, justamente devido à necessidade de 
aplicação de diferentes esforços ao longo do blank, o que pode causar distorções no cordão de solda $[4,5]$.

Uma das maneiras de se contornar tal efeito é aumentar o controle do processo produtivo e modificar o ferramental, de forma que o mesmo trabalhe com aplicação de forças distribuídas de forma a acompanhar as variações de espessura e de geometria do blank [6]. Trabalha-se também com o processo de Tailor Rolled Blank, o qual dispensa o processo de soldagem [5]. Porém, o TWB ainda é mais amplamente utilizado, pois seu ferramental é menos complexo e sua versatilidade é maior (pois se pode trabalhar com materiais que, embora apresentem a mesma espessura, possuam propriedades mecânicas e/ou químicas distintas) [1].

As principais aplicações, no veículo, de componentes obtidos a partir chapas confeccionadas por meio do Tailor Welded Blank são, segundo Kumar \& Reddy [2]: Painel interno de portas laterais; componentes estruturais da carroceria; longarinas; pilar "B" (Pilar central).

Para emprego por parte das indústrias automobilísticas são citados dois pontos principais: o processo de TWB, quando empregado em componentes de reforço estrutural, pode gerar redução de massa da ordem de até 16\% [6]; em alguns casos, embora não se consiga diretamente redução de massa, diminui-se o número de subcomponentes e a necessidade de inclusão de reforços. Com isso, reduz-se a complexidade do processo produtivo e se ganha em produtividade [7].

Apesar das referidas vantagens e da versatilidade do processo, deve-se atentar para o processo de soldagem. Isso porque o fluxo do material e sua conformabilidade ao longo do processo de estampagem são grandemente afetados pela orientação do cordão de solda. Quando dois metais de espessura distinta são soldados, os requisitos para conformação dos mesmos e a resistência mecânica obtida podem ser diferentes ao longo do blank confeccionado [1].

Dessa forma, a soldabilidade e a conformabilidade são dois conceitos que devem ser levados em consideração [7]. A influência do posicionamento do cordão de solda e da chamada razão de espessura (razão entre a maior e a menor espessura das chapas que compõe o blank) são frequentemente avaliadas, levando-se em consideração seus efeitos na conformação dos componentes, por meio das curvas limites de conformação [3].

Para garantir controle efetivo do processo também são utilizados softwares de simulação computacional, sendo o método mais empregado o de elementos finitos (Finite Element Analysis - FEA). Os mesmos auxiliam na previsão do comportamento do TWB durante o processo de estampagem, focando-se em prováveis falhas relacionadas ao processo de soldagem (em geral trabalha-se com um malha mais refinada na região do cordão do solda) $[1,4,5]$. Os modelos, embora cada vez mais precisos, precisam ser alimentados com dados relativos ao processo para apresentarem ainda maior correlação com a realidade.

Dentre as diversas maneiras de avaliar a influência das variáveis do processo de soldagem na conformabilidade e nas propriedades mecânicas do blank, destacamse: ensaios de tração; ensaios de embutimento e microdureza ao longo do blank $[3,7]$. Dessa forma, o estudo do processo TWB, focado nas variáveis do processo de soldagem e nas operações posteriores de conformação é crescente, focado na otimização e difusão da tecnologia.

Inicialmente o processo de Tailor Welded Blank apresentava elevados custos, o que inviabilizava a aplicação do mesmo em larga escala. Avanços recentes na tecnologia da soldagem e também da conformação, além do surgimento de novos materiais 
possibilitaram conciliar boa relação custo benefício, precisão dimensional e elevada resistência mecânica, mesmo nas proximidades do cordão de solda $[1,4]$.

Um dos focos da indústria automobilística é a redução do nível de emissões de poluentes e do consumo de veículos. Para isso tem-se o desafio de se trabalhar com materiais que sejam simultaneamente mais leves e resistentes mecanicamente. Nesse contexto o processo de TWB expande-se e ganha destaque, pois permite que se reduza o número de reforços empregados no veículo, bem como a necessidade de um maior número de etapas de manufatura [6].

Indústrias automobilísticas dos Estados Unidos e do Japão tem investido na pesquisa e utilização do processo de Tailor Blank, mais especificamente em peças que são soldadas a laser e também pelo processo denominado Upset Welding. Com isso, além de se reduzir a massa dos componentes do veículo, consegue-se também reduzir a quantidade de material necessário à fabricação de peças e componentes [7].

As pesquisas são conduzidas no sentido de se determinar qual o processo de soldagem mais adequado para união das chapas que irão compor o blank; os efeitos da zona termicamente afetada (ZTA) na conformabilidade do blank; o sentido mais adequado para o cordão de solda (paralelo ou transversal à linha de aplicação dos esforços mecânicos de conformação), dentre outros [3,7].

Dessa forma, o estudo do processo de obtenção de chapas por Tailor Welded Blank e das variáveis que influenciam no mesmo é de fundamental importância. Isso porque se permite a difusão da tecnologia, principalmente na indústria automobilística. Foca-se na utilização do material mais adequado e do melhor método de soldagem a ser empregado, garantindo-se maior controle das etapas de produção. Consegue-se então, por meio do TWB, conciliar premissas que eram, até então, divergentes: redução de massa e incremento de desempenho estrutura [6].

\section{MATERIAIS E MÉTODOS}

Os ensaios foram realizados a partir de chapas obtidas por meio do processo de Tailor Welded Blank. Para confecção do blank optou-se por utilizar o processo de soldagem a laser. Foram utilizados os seguintes materiais:

- Chapa 1: Aço CSN FEP05 (Intersticial Free - Livre de intersticiais)

- Espessura: $0,65 \mathrm{~mm}$

- Limite de escoamento (LE): $157 \mathrm{MPa}$

- Limite de Resistência à Tração (LRT): 284MPa

- Chapa 2: Aço CSN FEE 210 (Intersticial Free - Livre de intersticiais)

$\circ$ Espessura: $1,10 \mathrm{~mm}$

- Limite de escoamento: $217 \mathrm{MPa}$

- Limite de Resistência à Tração: $346 \mathrm{MPa}$

Trabalhou-se com a seleção de chapas por amostragem, partindo-se de fardos como os que se apresentam na Figura 2. 

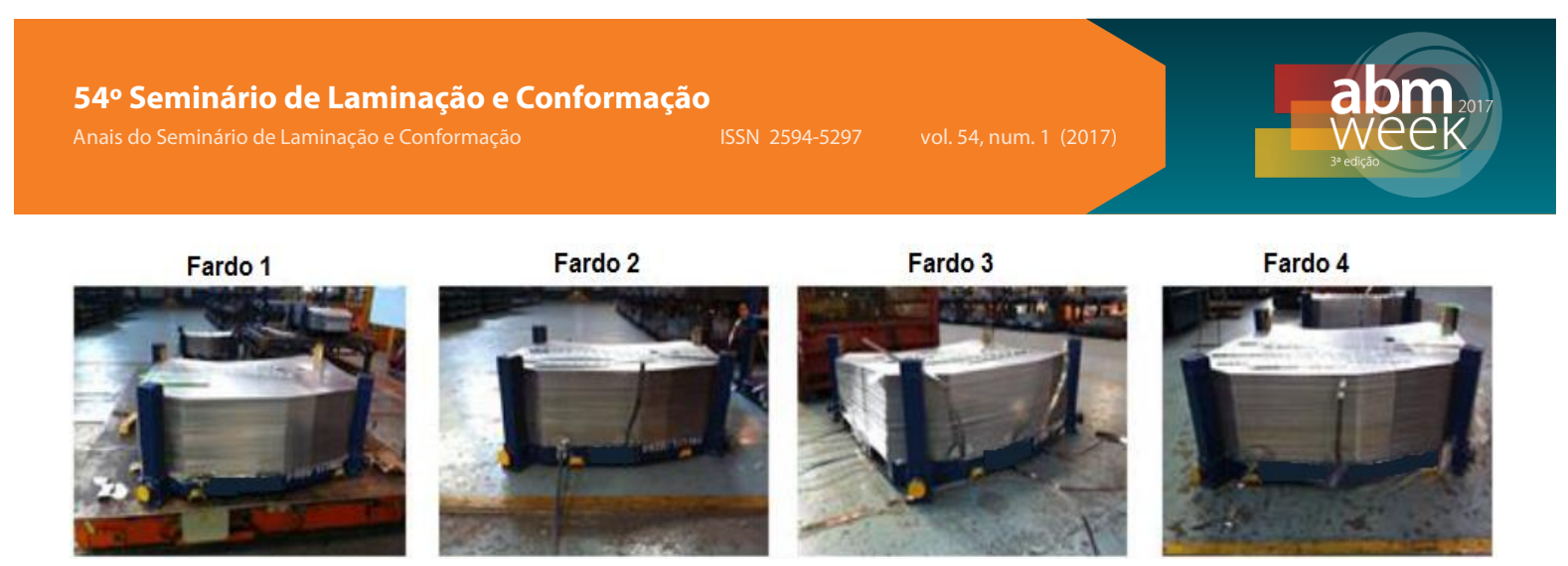

Figura 2. Fardos utilizados para seleção de amostras de chapas para teste

Para caracterização mecânica e análise de falhas das chapas estampadas foram realizados os seguintes ensaios:

\subsection{Ensaio de Embutimento pelo método Erichsen}

Para realização dos ensaios de embutimento optou-se pelo método Erichsen. Baseou-se nos procedimentos da NBR 16281:2014 (Determinação do Índice de Embutimento em Chapas de Aço Pelo Método Erichsen Modificado). Para tal, utilizou-se uma máquina ERICHSEN ${ }^{\circledR}$ modelo 142-40. Foram feitos ensaios em três amostras de chapas por fardo, as quais foram avaliadas ao longo de toda a linha de solda, conforme se apresenta na Figura 3.
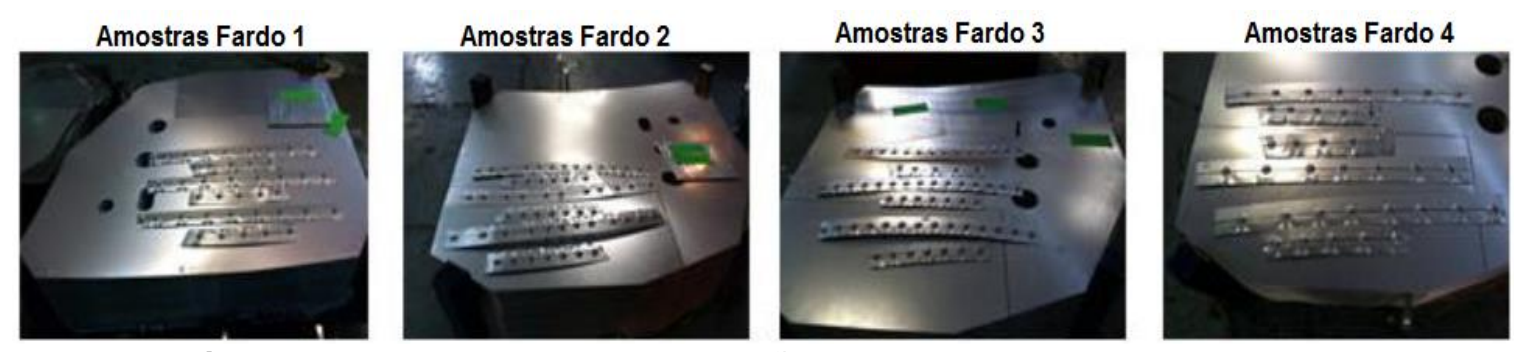

Figura 3. Fardos utilizados para seleção de amostras de chapas para teste

\subsection{Ensaio de Tração Uniaxial}

A fim de verificar o comportamento mecânico e determinar a provável região de falha dos blanks em caso de ruptura, foram realizados ensaios de tração uniaxial. Não foram levantados dados de deformação ou de tensão aplicados nos corpos de prova, pois o intuito do ensaio era qualitativo e não quantitativo.

Procedeu-se de acordo com a norma internacional da Sociedade Americana de Ensaios e Materiais (American Society for Testing and Materials) - ASTM E8, com taxa de deformação de $0,001 \mathrm{~s}^{-1}$. Os ensaios foram conduzidos em uma máquina de ensaios universais Instron ${ }^{\circledR} 5582$.

\subsection{Ensaios metalográficos}

A fim de determinar a região de ocorrência e o tipo de fratura encontrado nos corpos de prova de tração foram realizados ensaios de microscopia óptica (MO) e de microscopia de varredura por feixe de elétrons (MEV).

Para realização da $\mathrm{MO}$ as amostras foram previamente embutidas em baquelite. Após o embutimento procedeu-se com o lixamento em lixas \#200, \#400, \#600 e \#800. Utilizou-se alumina para o polimento e reagente $\mathrm{Nital}^{\circledR}{ }^{\circledR} 10 \%$ para 0 ataque químico. 


\section{RESULTADOS E DISCUSSÃO}

As marcas obtidas nos ensaios de embutimento do tipo Erichsen são apresentadas na Figura 4.
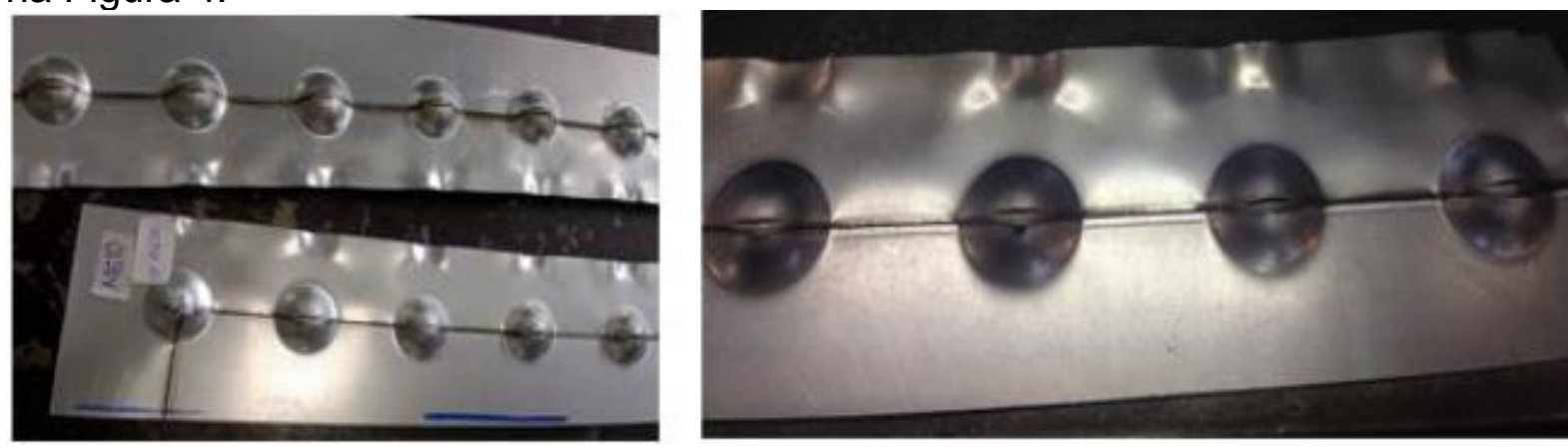

Figura 4. Marcas obtidas no ensaios de embutimento do tipo Erichsen, para duas amostras de diferentes fardos testados

Pode-se verificar que o rompimento da chapa não ocorre nem na chapa mais fina, nem na mais espessa. Há falha justamente na zona termicamente afetada (ZTA), o que não era esperado, uma vez que no processe de soldagem a laser essa região é muito estreita [8]. Em função dos materiais adotados e da diferença de espessura, a região mais propícia para falha deveria ser a chapa de menor espessura $[3,4,7]$. Nota-se ainda que esse comportamento não foi aleatório, sendo notado ao longo de toda a linha de testes.

Isso condiz com o observado pela regra das misturas [9] nem com o observado por Chen et al [3,10] e por Ahmetoglu et al [4]. Na Figura 5 apresentam-se os resultados obtidos para os ensaios de embutimento realizados em lados opostos de blanks, em chapas já preparadas para estampagem das peças finais.

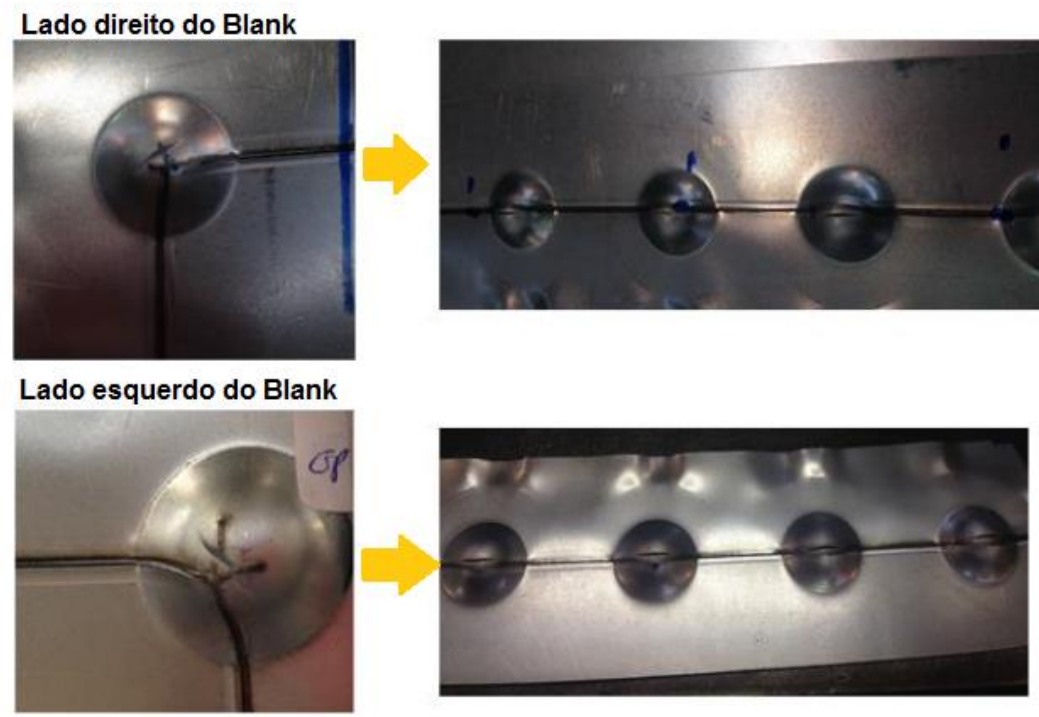

Figura 5. Resultados obtidos para o ensaio de embutimento em lados opostos de blanks já preparados para estampagem final das peças

Percebe-se que tanto no lado direito quanto no esquerdo do blank a falha se inicia na ZTA e se propaga a partir dessa, seja de forma radial ou transversal. Isso indica que durante as operações de estampagem profunda pode haver rasgamento das chapas, conforme apontado por Merklein et al [1] e HIRCZA-MICHALSKA, ROJEK e FRUITOS [6]. 
Na Figura 6 apresentam-se os corpos de prova de tração após realização do ensaio.

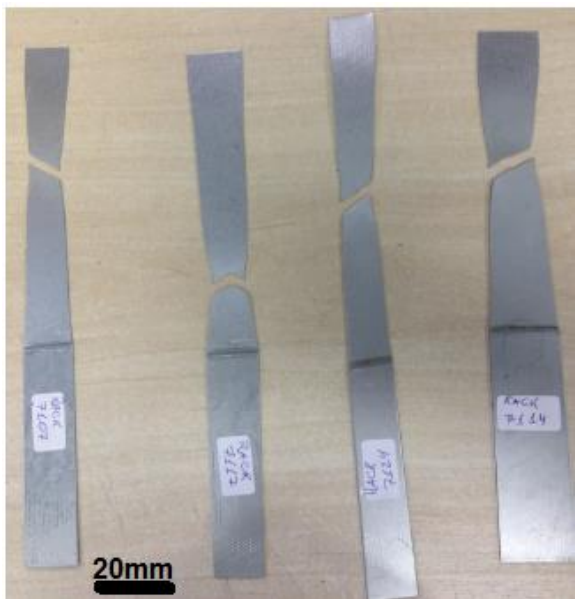

Figura 6. Corpos de prova de tração uniaxial após realização do ensaio

Os ensaios de tração, apesar de padronizados pela ASTM e outras instituições normativas, podem ser executados de diferentes formas, a depender do objeto de análise e dos resultados e dados que se espera obter. Especificamente para 0 processo de TWB, não há consenso quanto ao posicionamento e sentido do cordão de solda, nem da razão de espessura das chapas $[1,3,5,7,9,10]$.

Esse fato é ainda mais evidente quando se comparam os resultados obtidos nos ensaios de embutimento com a região de fratura encontrada nos corpos de prova de tração. Evidentemente que a instabilidade plástica e a fratura tendem a ser favorecidas em regiões chamadas concentradoras de tensão [11,12], o que poderia explicar a ruptura justamente na chapa de menor espessura, em todos os corpos de prova avaliados.

Esse resultado é condizente com o encontrado por MIN, KIM e KANG [7] e HIRCZAMICHALSKA, ROJEK e FRUITOS [6]. Justamente por esse motivo, algumas referências $[1,2,3,10]$ defendem a realização de ensaios de tração com corpos de prova cujo cordão de solda seja posicionado longitudinalmente.

$\mathrm{Na}$ Figura 7 apresentam-se as superfícies de fratura da chapa de um dos fardos testados, na região de embutimento. As imagens foram obtidas com auxílio de MEV.

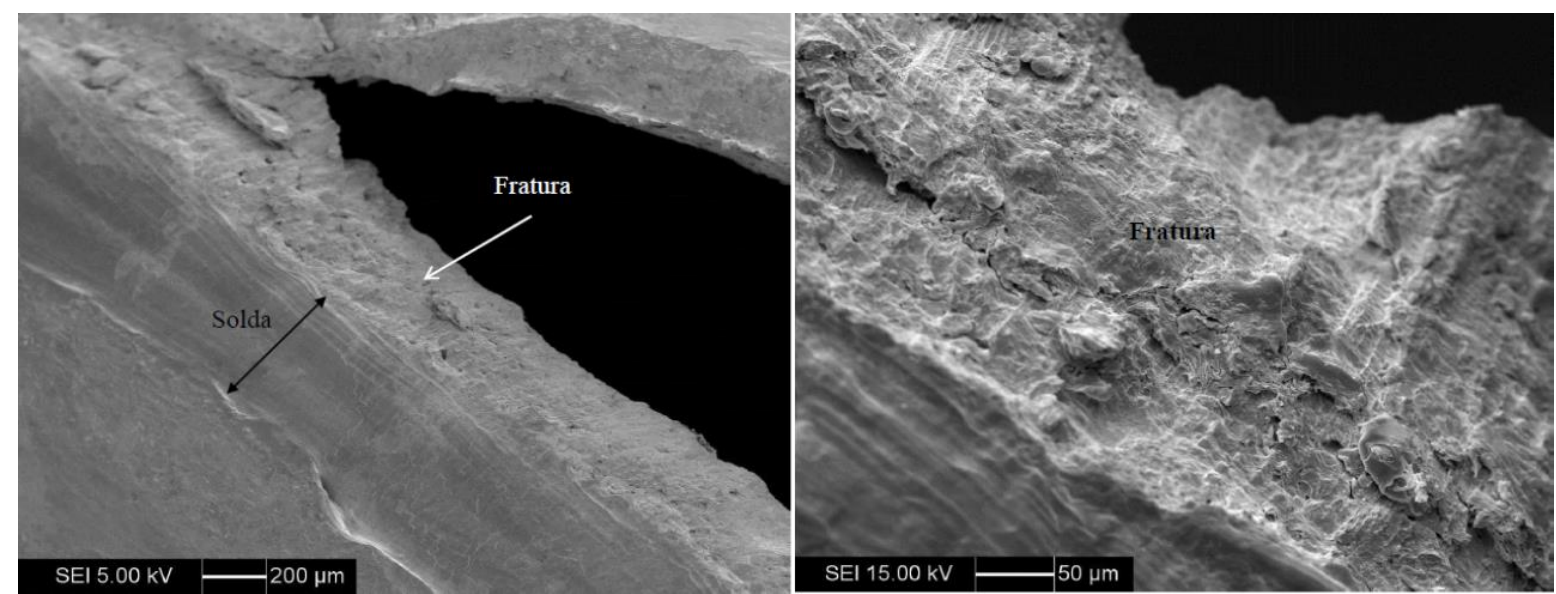

Figura 7. Superfícies de fratura da chapa de um dos fardos testados, na região de embutimento.

Percebe-se que a fratura ocorre justamente na ZTA, bem ao lado da solda. A propagação da falha, nessa chapa, ocorreu paralelamente à solda. Há tanto 
superfícies que aparentam-se com facetas de clivagem (indicando certa fragilidade do material e da ZTA) quanto microcavidades (o que já indicaria regiões, ainda que próximas da solda, com maior tenacidade). Esses resultados condizem com o que se espera do perfil de microdureza de chapas obtidas por TWB, nas quais a região soldada tende a ser mais dura e frágil, independente da espessura das chapas envolvidas $[3,9,10]$.

$\mathrm{Na}$ Figura 8 apresenta-se a análise metalográfica, feita por meio de $\mathrm{MO}$, de um dos corpos de prova de tração, após a fratura.

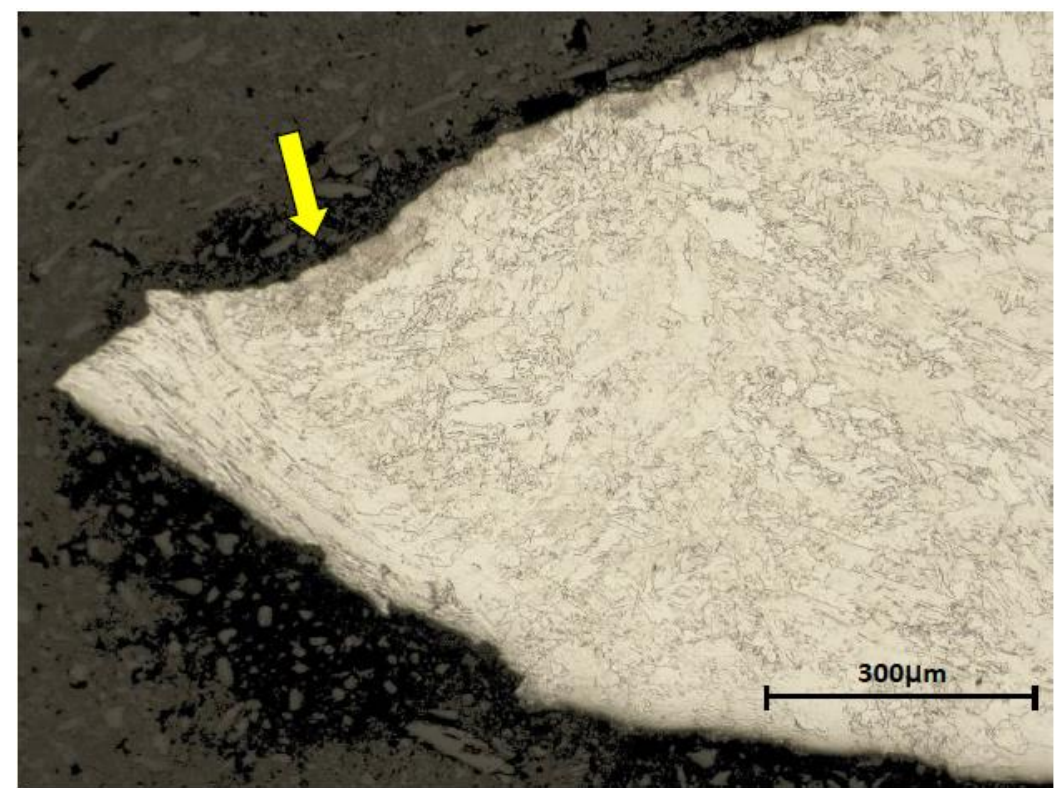

Figura 8. Análise metalográfica de um dos corpos de prova de tração após a ruptura

A seta amarela delimita a região entre o embutimento e a amostra avaliada. As análises feitas com auxílio da MO foram inconclusivas, uma vez que todos os corpos de prova de tração fraturaram na região da chapa de menor espessura, bem distante da região soldada e da ZTA.

\section{CONCLUSÃo}

- A fratura da solda ocorreu na zona termicamente afetada (ZTA);

- A fratura ocorreu por esforços de tração simples, gerados pelo processo de estampagem do componente;

- O local da fratura, na ZTA da chapa de menor espessura, é o local esperado para este tipo de evento, devido à alteração de dureza gerada pelo aquecimento do processo de soldagem e a menor espessura da chapa;

- Não foram encontradas não conformidades na região soldada que pudessem favorecer a ocorrência da falha;

- Os limites de conformação dos aços FEP05 0,65mm e FEE210 1,10mm se mostraram nos mesmos níveis, individualmente. O limite de conformação do conjunto soldado, entretanto, deve ser analisado e confrontado com as deformações obtidas neste artigo. 


\section{Agradecimentos}

Os autores agradecem à FIAT Chrysler Automóveis do Brasil LTDA pelos dados e apoio técnico fornecidos.

\section{REFERÊNCIAS}

1 MERKLEIN, Marion et al. A review on tailored blanks: Production, applications and evaluation. Journal Of Materials Processing Technology. Erlangen, p. 151-164. set. 2013.

2 KUMAR, T. Kishore; REDDY, K. Jayasimha. Taylor welded blank. ljret: internacional Journal of Research in Engineering and Technology. Andra Pradexe, p. 63-72. abr. 2015.

3 CHAN, S. M.; CHAN, L. C.; LEE, T. C.. Tailor-welded blanks of diferent thickness ratios effects on forming limit diagrams.Journal Of Materials Processing Technology. Hong Kong, p. 95-101. nov. 2002.

4 AHMETOGLU, Mustafa A. et al. Deep drawing of round cups from tailor-welded blanks. Journal Of Materials Processing Technology. Ohio, p. 684-694. mar. 1994.

5 TAN, C. J.; MORI, K.; ABE, Y.. Forming of tailor blanks having local thickening for control of wall thickness of stamped products. Journal Of Materials Processing Technology. Toyohashi, p. 443-449. out. 2007

6 HIRCZA-MICHALSKA, M.; ROJEK, J.; FRUITOS, O.. Numerical simulation of car body elements pressing applying tailor welded blanks: pratical verification of results. Archives Of Civil And Mechanical Engineering, Gliwice, v. 10, n. 4, p.31-44, jan. 2010.

$7 \quad$ MIN, K. B.; KIM, K. S.; KANG, S. S.. A study on resistance welding in steel sheets using a tailor-welded blank (1st report): Evaluation of upset weldability and formability. Journal Of Materials Processing Technology. Pusan, p. 186-192. out. 1998.

8 MARQUES, P. V. MODENESI, P. J. BRACARENSE, A. Q., 2009, "Soldagem: Fundamentos e Tecnologia".3 ed. Belo Horizonte: Editora UFMG, 2009. 363p.

9 ABDULLAH, K.; WILD, P.P.; JESWIET, J.J; GHASEMPOOR, A.. Tensile test for weld deformation properties in similar gage tailor welded blanks using the rule of the mixtures. Journal of Materials Processing Technology, Kingston, v.1, n. 112, p.91-97, jan. 2001.

10 CHAN, L. C.; CHAN, S.M.; CHENG, C.H.; LEE, T.C.. Formability and Weld Zone Analysis of Tailor-Welded Blanks for Various Thickness Ratios. Journal Of Engineering Materials And Technology, [s.I.], v. 127, n. 2, p.179-185, 2005. ASME International.

11 CALLISTER JUNIOR, William D.. Ciência e Engenharia de Materiais: uma introdução. 7. ed. Rio de Janeiro: LTC, 2008.

12 DIETER, George E.. Metalurgia Mecânica. 2. ed. Rio de Janeiro: Guanabara Dois, $1981.660 \mathrm{p}$ 